\title{
MolMap \\ Visualizing Molecule Libraries as Topographic Maps
}

\author{
Martin Gronemann ${ }^{1}$, Michael Jünger ${ }^{1}$, Nils Kriege ${ }^{2}$ and Petra Mutzel ${ }^{2}$ \\ ${ }^{1}$ Institut für Informatik, Universität zu Köln, Germany \\ ${ }^{2}$ Dept. of Computer Science, Technische Universität Dortmund, Germany \\ \{gronemann, mjuenger\}@informatik.uni-koeln.de, \{nils.kriege, petra.mutzel\}@tu-dortmund.de
}

Keywords: Graph Drawing, Clustered Graphs, Topographic Maps, Drug Discovery, Molecule Libraries

\begin{abstract}
We present a new application for graph drawing and visualization in the context of drug discovery. Combining the scaffold-based cluster hierarchy with molecular similarity graphs — both standard concepts in cheminformatics - allows one to get new insights for analyzing large molecule libraries. The derived clustered graphs represent different aspects of structural similarity. We suggest visualizing them as topographic maps. Since the cluster hierarchy does not reflect the underlying graph structure as in (Gronemann and Jünger, 2012), we suggest a new partitioning algorithm that takes the edges of the graph into account. Experiments show that the new algorithm leads to significant improvements in terms of the edge lengths in the obtained drawings.
\end{abstract}

\section{INTRODUCTION}

Drug discovery is a tedious and expensive process that typically involves the experimental evaluation of large compound libraries by high-throughput screening in order to identify promising small molecules that bind to a specific biological target. These compounds may serve as a starting point for chemical modifications, e.g., in order to reduce side effects or to improve potency. A guiding principle during this process is to elucidate and exploit the relationship between the structure of a molecule and its biological properties.

In the course of the drug discovery process typically structural information on compounds as well as experimental results are generated. Supporting the drug discovery process based on this data by adequate computational methods like, e.g., largely automated data mining algorithms, is a fundamental task in cheminformatics (Brown, 2009). However, only recently several sophisticated visual analysis tools for this domain have been developed (Wetzel et al., 2009; Klein et al., 2012; Lounkine et al., 2010; Strobelt et al., 2012; Herhaus et al., 2009) with the aim to allow the user to explore the chemical space and make decisions based on his expert knowledge. These tools often represent sets of molecules and their structural relations by means of graphs, and they rely on graph drawing techniques for visualization. Since structural relations are rather complex, different approaches have been developed, some of which orga- nize molecules in a tree-like hierarchy while others are based on pairwise similarities. The complexity of structural relations, the amount of data and the different perspectives chemists from different branches of chemistry may have makes it difficult to find an approach that satisfies all needs.

Irwin recently summarized the problem as follows: "In principle, one would like to be able to organize and browse large chemical datasets [...] as easily as one can today browse maps on the internet." (Irwin, 2009) This comparison gives the impetus for our approach to represent molecule sets by clustered graphs, which are then visualized as topographic maps based on a method recently proposed (Gronemann and Jünger, 2012). The use of clustered graphs allows us to model different aspects of structural similarity between compounds in a single graph and to visualize them in one picture in which similar molecules appear close to each other. Clustered graphs have not yet been applied to visualize chemical compounds, the most likely reason is that drawing them is difficult. This is caused by the requirement to generate an appealing drawing of the underlying graph that simultaneously represents the hierarchy of nested subsets of its nodes. The issue becomes especially challenging when the cluster hierarchy does not reflect the "natural" clusters of the underlying graph. This problem arises with our representation of molecular data sets by clustered graphs and was not addressed in (Gronemann and Jünger, 
2012). Therefore we develop a new partitioning procedure that exploits the degrees of freedom in the algorithm with the goal to obtain shorter edges. In order to meet the requirements of the application domain, our procedure also supports the display of structural formulae of compounds and the superimposition of a heat map that allows the representation of property values.

\section{RELATED WORK}

A straight-forward and wide-spread approach to browse compound datasets are molecular spreadsheets depicting compounds and their properties in table form. Recently several more sophisticated tools for the visualization of compound sets have been developed. Many of these represent chemical compounds by means of graphs:

In (Wawer et al., 2008) the concept of 'Networklike Similarity Graphs' was introduced to study structure-activity relationships of small molecules. Here, compounds are represented by nodes and two nodes are connected by an edge if their compounds exceed a certain similarity threshold. These graphs are then visualized using a force-directed layout algorithm (Fruchterman and Reingold, 1991). In addition, similarities are used to derive a hierarchical clustering of all nodes. However, for visualization only a single clustering is selected instead of displaying the full hierarchy. The approach was implemented by the open-source application SARANEA (Lounkine et al., 2010) based on the framework JUNG ${ }^{1}$. A different application, also based on pairwise molecular similarities, is HiTSEE (Strobelt et al., 2012). This tool maps compounds on the plane trying to preserve distances derived from similarities by Multidimensional Scaling (MDS).

Several concepts to organize compounds via hierarchical classification schemes have been proposed (Schuffenhauer and Varin, 2011). These are, e.g., based on clustering algorithms or on domain specific methods based on rules incorporating chemical knowledge. An approach of the second category is the so-called scaffold tree (Schuffenhauer et al., 2007) on which the visual analysis tool Scaffold Hunter (Wetzel et al., 2009; Klein et al., 2012) is based. For visualization, a radial style tree drawing algorithm is used. The tool MolWind (Herhaus et al., 2009) is also based on the scaffold tree concept, but uses NASA's World Wind engine to map scaffolds to a virtual globe. While MolWind is not directly related

\footnotetext{
$1_{\text {http: / / jung. sourceforge. net }}$
}

to graph drawing, we take up the MolWind idea to map chemical information to intuitively understandable geographic representations, and realize it in the realm of clustered graph drawing.

While clustered graph drawing is a lively research topic in graph drawing (Sugiyama and Misue, 1991; Huang and Eades, 1998) (see, e.g., in GDEA ${ }^{2}$ ), only few approaches exist for drawing clustered graphs as maps. Gansner et al. have suggested the use of geographic maps for enhancing clusters and visualizing similarities (Gansner et al., 2009; Gansner et al., 2010). They first compute a partition of the nodes by applying a cluster algorithm, then use a force-directed method or MDS to compute a layout, and finally use both to create a colored map. The combination of the layout and clustering methods used for generating the map, plays an important role regarding the visual shape of the clusters in the final layout. In order to obtain a map where the countries form compact areas, requires that the result produced by the layout algorithm matches the partitioning of the clustering step. Otherwise, the partitions are visually scattered, this problem is referred to as fragmentation.

An alternative approach for visualizing hierarchically clustered graphs as topographic maps has been suggested recently in (Gronemann and Jünger, 2012). Topographic maps have a long history in information visualization. Mostly used for visualizing point distributions, they offer a natural way to explore data.

In (Fabrikant et al., 2010) the effect of using topographic maps as metaphor is studied. They confirm the usage of elevation levels as an effective way to encode similarities. Gronemann and Jünger propose to represent the complete hierarchy of a clustered graph by elevation levels. The elevation model is defined such that nodes in different subtrees are separated by a valley. In other words, nodes in the same cluster are placed together on a plateau. This simple idea requires that the layout algorithm generates compact areas for all clusters.

By using a treemap approach for the layout the problem of fragmentation is avoided. Several methods for generating treemaps exist in the literature. Starting with the work of (Johnson and Shneiderman, 1991), where a hierarchy is mapped on a space filling curve, most algorithms are based on a rectangular subdivision scheme. A good overview over the vast amount of treemap techniques can be found in (Schulz, 2011).

\footnotetext{
${ }^{2}$ Graph Drawing E-Print Archive, http://gdea. informatik.uni-koeln.de/
} 


\section{COMPOUND LIBRARIES AND CLUSTERED GRAPHS}

A clustered graph is a tuple $(G, T)$, where $G=(V, E)$ is graph and $T=\left(V_{T}, E_{T}\right)$ is a rooted tree such that the leaves of $T$ are the nodes in $V$. Each inner node $v$ of $T$ corresponds to a cluster of $G$ comprising all leaves of the subtree rooted at $v$. Therefore the inner nodes of $T$ are called cluster nodes, $T$ is referred to as cluster tree and defines a hierarchy of nested subsets of $V$.

Approaches to explore chemical compound libraries typically allow the user to navigate between regions of molecules with a similar structure. We combine two orthogonal concepts from cheminformatics to derive clustered graphs that represent two different aspects of structural similarity.

\subsection{Molecular Similarity Graph}

A set of compounds with pairwise similarities can be represented by a graph: Molecules are associated with nodes and two nodes are connected by an edge if their similarity exceeds a certain threshold. This approach is applied in, e.g., (Wawer et al., 2008; Lounkine et al., 2010). We additionally annotate edges with the corresponding similarity values. For a given set of molecules $M$ we define a similarity graph $G(M)=(V, E)$ with edge weights $w$ according to $V=M, E=\{(u, v) \in V \times V \mid \operatorname{sim}(u, v) \geq t\}$, and $w((u, v))=\operatorname{sim}(u, v)$ for all $(u, v) \in E$, where $\operatorname{sim}(\cdot, \cdot)$ is a similarity measure defined on molecules and $t$ is a threshold value with direct influence on the density of the graph.

While computing adequate structural similarity measures for molecules is a wide and challenging research topic on its own (Maggiora and Shanmugasundaram, 2011), we rely on standard techniques often used in practice: Molecules are represented by so-called fingerprints, i.e., binary vectors of constant size, where bits encode the presence or absence of certain fragments (Brown, 2009). We employ the pathbased hash-key fingerprint provided by the cheminformatics toolkit CDK (Steinbeck et al., 2006). The fingerprints are then compared with each other by computing the Tanimoto coefficient in order to obtain pairwise similarities.

\subsection{Scaffold-based Cluster Hierarchy}

A different approach to organizing chemical compound sets is based on a hierarchical classification scheme representing molecules by their core structures referred to as scaffolds. The scaffold tree al- gorithm (Schuffenhauer et al., 2007) builds a tree-like hierarchy of scaffolds. It essentially proceeds as follows: For each molecule in the dataset its scaffold is created by pruning all terminal side chains. Then the scaffold is successively simplified by removing a single ring in each step such that the resulting parent scaffold stays connected. The procedure stops when a one-ring scaffold is obtained. Since in each step multiple rings can be removed, a unique parent scaffold is selected by a set of rules incorporating chemical knowledge with the aim to preserve the most characteristic ring structure. When the procedure is applied to a set of molecules, typically some have the same scaffold and several scaffolds share the same parent scaffold. A scaffold tree is obtained by merging these multiple scaffolds and connecting all one-ring scaffolds to a virtual root. Figure 1 shows four scaffolds and their relation in a scaffold tree branch.

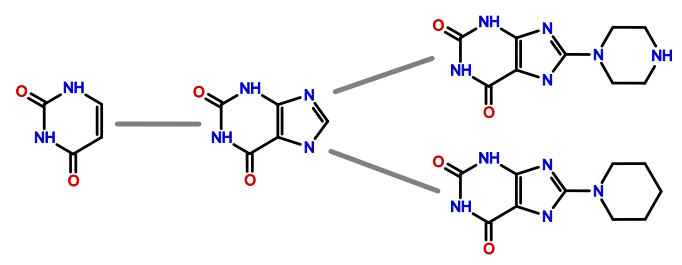

Figure 1: A branch of a scaffold tree.

Generating the scaffold tree for a set of molecules $M$ and connecting all molecules to their scaffold yields a valid cluster tree for the similarity graph $G(M)$. While the scaffold tree concept has been successfully applied to various research tasks (Wetzel et al., 2009; Bon and Waldmann, 2010), an obvious conceptual weakness is that side chains are not taken into account although they may constitute key functional groups (Irwin, 2009). Therefore, the combination with a similarity graph based on a wholemolecule structural similarity measure may help to alleviate this drawback.

\section{EDGE-AWARE DRAWING OF CLUSTERED GRAPHS}

Visualizing the hierarchy provided by the scaffold tree and drawing edges of the similarity graph at the same time turned out to be a challenge. The main problem is that the hierarchy is not related to the similarity graph, because nodes contained in a cluster are not necessarily similar. When the original method proposed in (Gronemann and Jünger, 2012) is applied to these graphs, the results are rather unpleasant. Especially on very sparse graphs, edge clutter occurs. In (Gronemann and Jünger, 2012), where a clustering 


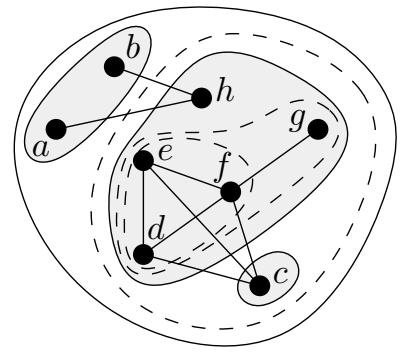

(a) Clustered Graph

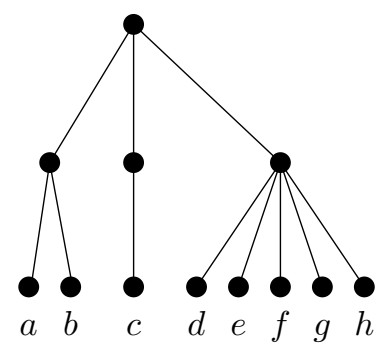

(b) Input Cluster Tree

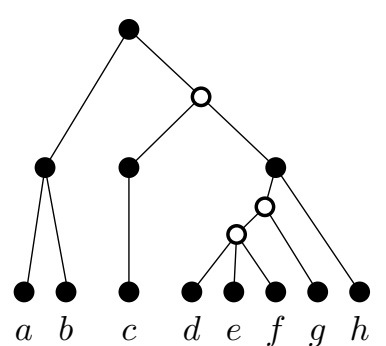

(c) Extended Cluster Tree

Figure 2: Example of a clustered graph (a) with cluster tree (b). The extended cluster tree (c) contains dummy nodes determined by betweenness based clustering. Dashed shapes in (a) depict the clusters representing these dummy nodes.

method is applied to the input, and thus the clusters are consistent with the placement algorithm, nodes that are well connected are placed close to each other, while nodes with higher graph theoretic distance are located in different subtrees. Before we present two improvements, we sketch the outline of the original method. First, the input graph is clustered using the algorithm of (Girvan and Newman, 2002) to obtain a cluster hierarchy with the aforementioned properties. Then a tree mapping approach called fat polygon partitioning, based on (de Berg et al., 2010), is used. The nodes are placed inside their partition that form a nested structure of convex polygons representing the hierarchy. The layout is then transformed into a triangle mesh by applying a Delaunay triangulation. This mesh serves both as a basis for drawing a topographic map and as a routing network for edge bundling. For more details, see (Gronemann and Jünger, 2012).

In the following we present two improvements of the original method. First, we suggest to extend the input hierarchy by adding virtual clusters without changing the nested structure of the input. These new clusters help to place highly connected groups of nodes together. Second, the fat polygon partitioning is modified to optimize edge length and to place clusters based on an arbitrary edge set, while still generating good polygons. The goal is to make the tree map algorithm aware of the edges and exploit the degrees of freedom offered by the input hierarchy and the polygon partitioning. Finally, we evaluate the modifications based on the instances of our application area.

\subsection{Extending the Input Hierarchy}

The inner nodes of the scaffold tree usually contain many children. In this case the fat polygon partition must create a binary subtree for the subdivision procedure. However, since the provided input hierarchy is not edge-aware, the subdivision procedure may result in edge clutter. This is due to the fact that highly connected child clusters are placed in distant subtrees.
In order to solve this problem, we use edge betweenness clustering to extend the input hierarchy. The algorithm proposed in (Girvan and Newman, 2002) is rather simple. For all edges, the betweenness score is calculated and the edges with the highest score are removed. This procedure is repeated until the graph becomes disconnected. The connected components serve as clusters on which the method recurses.

This method can easily be adapted to work on an existing hierarchy. We traverse the input hierarchy top-down. For each cluster $C$ with children $C_{1}, \ldots, C_{n}$, $n>2$, we construct a graph $G(C)$ induced by the underlying edge set $E$ and the children of $C$. Let $V\left(C_{i}\right)$ denote the graph nodes contained in the subtree rooted at $C_{i}$. Let $E\left(C_{i}, C_{j}\right)=E \cap\left(V\left(C_{i}\right) \times V\left(C_{j}\right)\right)$ denote the edges having one end point in $C_{i}$ and the other in $C_{j}$. The obvious way to obtain the inter-cluster edge weights $w\left(C_{i}, C_{j}\right)$ is to sum up the 'clusterparallel' edge weights according to

$$
w\left(C_{i}, C_{j}\right)=\sum_{e \in E\left(C_{i}, C_{j}\right)} w(e) .
$$

The original algorithm is then used to obtain a hierarchy for this graph, which we can insert into the existing subtree. The leaves of the obtained hierarchy correspond to the children of the cluster node $C$, which can be replaced by the root of the hierarchy.

In Figure 2 a small example is displayed. The dashed shapes are the newly inserted clusters after the algorithm has decomposed as much as possible without violating the nested structure of the input hierarchy. The newly created clusters are used as "ghost clusters", which are not visible on the final map. Their only purpose is to group tightly connected components together. In case that the result is not a binary tree, the transformation used in (Gronemann and Jünger, 2012) is applied. Notice that the extended hierarchy is based on intra-cluster adjacency only and does not take external edges into account. This issue is addressed in the following section. 


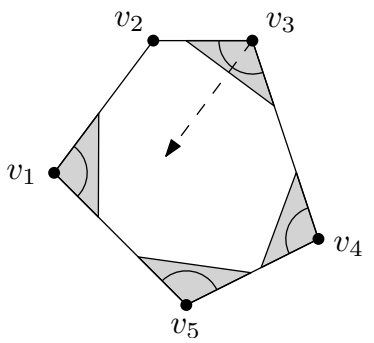

(a)

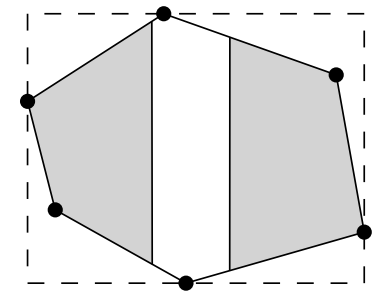

(b)

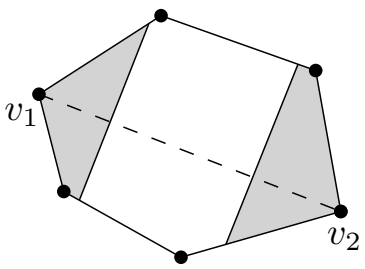

(c)

Figure 3: Enumeration of allowed cuts. In (a) we only want to cut off a small part of the polygon. The two choices when choosing an axis-aligned cut and a cut perpendicular to the diameter defined by $v_{1}, v_{2}$ are shown in (b) and (c), respectively.

\subsection{Edge-aware Polygon Partitioning}

In this section we investigate the degrees of freedom of the polygon partitioning and how to exploit them to minimize the length of edges between clusters.

A tree map based on fat polygon partitioning follows the same principle as most tree map algorithms. The idea is to recursively subdivide a convex polygon in order to obtain a nested structure of polygons representing the cluster tree. Starting with the root which represents the boundary polygon the algorithm proceeds in a top-down manner: At each cluster node, the associated polygon is split into two convex subpolygons which represent the subtrees rooted at the two children. The cutting line is chosen, such that the area of the subpolygons is proportional to the number of leaves in the corresponding subtrees. We place the graph nodes, i.e., the leaves of the tree, in the centroid of the associated polygon computed by the partitioning.

The basic problem in the individual steps of the partitioning algorithm can be summarized as follows: We are given a convex polygon $P$ with $k$ vertices and a parameter $0<a \leq \frac{1}{2}$, where $a$ is the fraction of the area we require for the smaller child. Let $\operatorname{area}(P)$ denote the area of $P$ and $\operatorname{diam}(P)$ its diameter, i.e., the maximum distance between two vertices of $P$. We want to find a direction for a cutting line that partitions $P$ into two subpolygons $P_{1}$ and $P_{2}$, such that $\operatorname{area}\left(P_{1}\right)=a \cdot \operatorname{area}(P)$ and $\operatorname{area}\left(P_{2}\right)=(1-a) \cdot \operatorname{area}(P)$. When given a cut direction, we choose the orientation of the cutting line perpendicular to this direction. In general finding such a cut is easy for a convex polygon. However, an advantageous characteristic of the greedy algorithm proposed in (de Berg et al., 2010) is that it yields polygons with bounded aspect ratios, where the aspect ratio is defined as $\operatorname{asp}(\mathrm{P})=\operatorname{diam}(P)^{2} / \operatorname{area}(P)$. We want to preserve this property without unnecessarily restricting the number of allowed cuts.

In (de Berg et al., 2010) two main cases are distinguished: In the first case, when we want to cut off a small piece, that is when $a \leq 1 / k^{2}$, the bisector at the vertex with the smallest interior angle is taken as the cut direction. However, the guarantee of the small aspect ratio only relies on the fact that the smallest interior angle of a convex polygon is bounded by $\pi \cdot\left(1-\frac{2}{k}\right)$ (de Berg et al., 2010). Thus, we are allowed to choose any other bisector as long as the interior angle at the corresponding vertex is small enough. In Figure 3(a) an example is given, where we are allowed to choose every bisector, except the one at $v_{2}$, which is not small enough.

In the second case, when $a>1 / k^{2}$, the aspect ratio of the boundary polygon must be considered. When $P$ has a good aspect ratio, i.e., $\operatorname{asp}(P) \leq k^{6}$, we are allowed to choose any direction for a cut (de Berg et al., 2010). In this case and when $a>\frac{1}{3}$ holds, our implementation chooses an axis-aligned cut normal to the longest side of the bounding rectangle for aesthetic reasons, see Figure 3(b). Otherwise, when $\operatorname{asp}(P)>k^{6}$ or $1 / k^{2}<a \leq \frac{1}{3}$ holds, we choose the direction of the line representing the diameter of the polygon. This is the line that connects any two vertices of $P$ with maximum distance to each other, $\mathrm{cf}$. Figure 3(c).

The key idea is to enumerate all allowed cuts for a given polygon and a parameter $a$ in each step of the partitioning procedure and to take the one that minimizes the edge length. That is, we try to place the polygon of a child cluster in the direction of clusters to which it is highly connected. Figure 4 illustrates

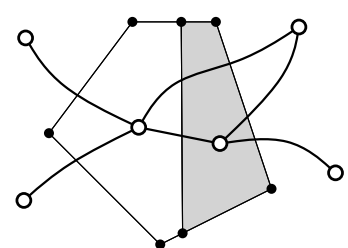

(a)

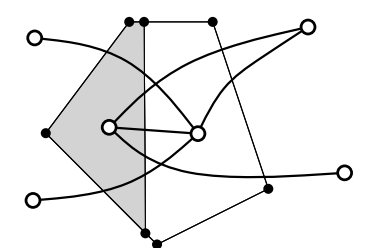

(b)
Figure 4: Example for two possible partitions of a polygon with adjacencies to external clusters. In (a) the total edge length is less compared to the partition in (b). 


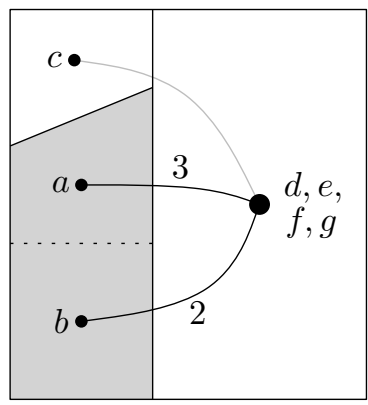

(a) Initial situation

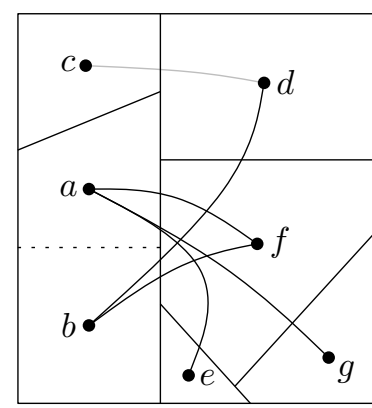

(b) Unbalanced refinement

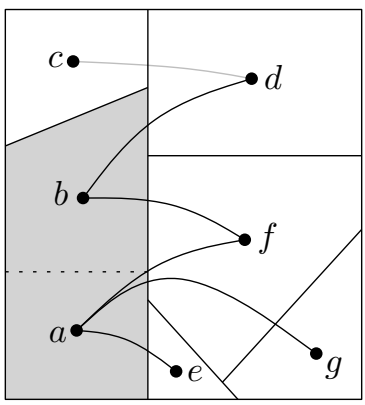

(c) Balanced refinement

Figure 5: Example of layouts obtained for different refinement strategies.

the idea. We will apply this procedure in a top down manner to obtain a partitioning that is optimized for edge length. However, there is no accurate information available as to where these adjacent clusters are located because the corresponding polygon has not been computed yet. Therefore, we propose a heuristic to decide which cut to use based on the partial knowledge of the surrounding clusters.

The available information on node positions depends on the current state of the subdivision process. While the cluster tree is typically traversed in a depth-first fashion, we propose a different procedure to achieve a more balanced level of refinement: The subdivision process always splits the polygon covering the larger area first. We keep all cluster nodes whose polygons still have to be partitioned in a priority queue $Q$ ordered by area requirement. These nodes, together with the leaves that have been processed, form a layer $L$ of nodes with associated polygons that we call active. The polygons of all active nodes cover the entire boundary polygon and realize the preliminary layout on which the selection of an allowed cut is based.

Figure 5 exemplifies the influence of the refinement order. When partitioning the gray polygon containing $a$ and $b$ in Figure 5(a) there is no accurate information available on the positions of $d, e, f, g$. Therefore, the polygon of $a$ is placed in the middle since minimizing the distance representing the length of three inter-cluster edges is prioritized. The result of this bad decision is displayed in Figure 5(b). When polygons are partitioned in order of decreasing size the information on neighboring polygons is more accurate. This typically results in a better final layout. Figure 5(c) shows the cut that would be chosen after the large right polygon has been refined.

We introduce additional notation to precisely define the criterion we use to assess the potential of a cut to minimize edge lengths. Let $C$ be a node of the cluster tree. We use $\operatorname{ctr}(C)$ to refer to the centroid of the associated polygon; awc $(C)$ denotes the average weighted center of the centroids of active polygons adjacent to $C$ weighted by inter-cluster edges, i.e.,

$$
\operatorname{awc}(C)=\frac{1}{w(C, L)} \sum_{P \in L} \operatorname{ctr}(P) \cdot w(C, P),
$$

where $w(C, L)=\sum_{P \in L} w(C, P)$. The value of awc $(C)$ can be considered as the preferred location of (the centroid of) the polygon associated with $C$.

Consider an active node $C \in Q$ with children $C_{1}$ and $C_{2}$. Each allowed cut assigns a subpolygon to the two children, cf. Figure 3. The idea is to choose the cut that minimizes the distance between the centroids of the subpolygons and their preferred positions according to the average weighted center. We define the distance between the two points associated with a node $C$ as

$\operatorname{dist}(C)= \begin{cases}\|\operatorname{awc}(C)-\operatorname{ctr}(C)\| & \text { if } w(C, L) \neq 0, \\ 0 & \text { otherwise. }\end{cases}$

Note that in the second case there are no edges leaving the cluster $C$ and the position of the polygon has no effect on edge lengths. We choose the cut that minimizes

$$
\sum_{i \in\{1,2\}} \operatorname{dist}\left(C_{i}\right) \cdot w\left(C_{i}, L\right)
$$

where the factor $w\left(C_{i}, L\right)$ reflects the impact of the individual children. Figure 6 illustrates the method for a given cut.

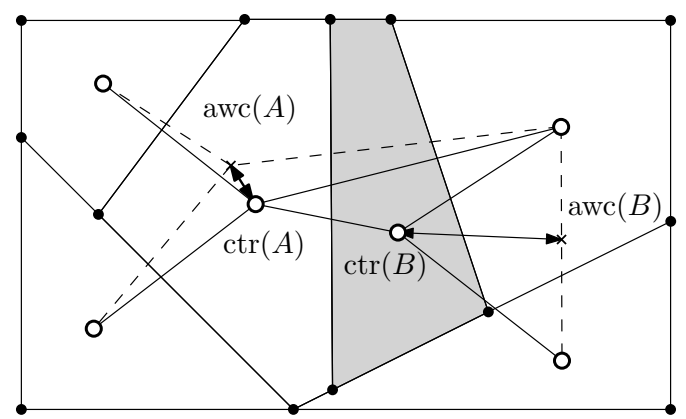

Figure 6: Edge-aware cut selection 


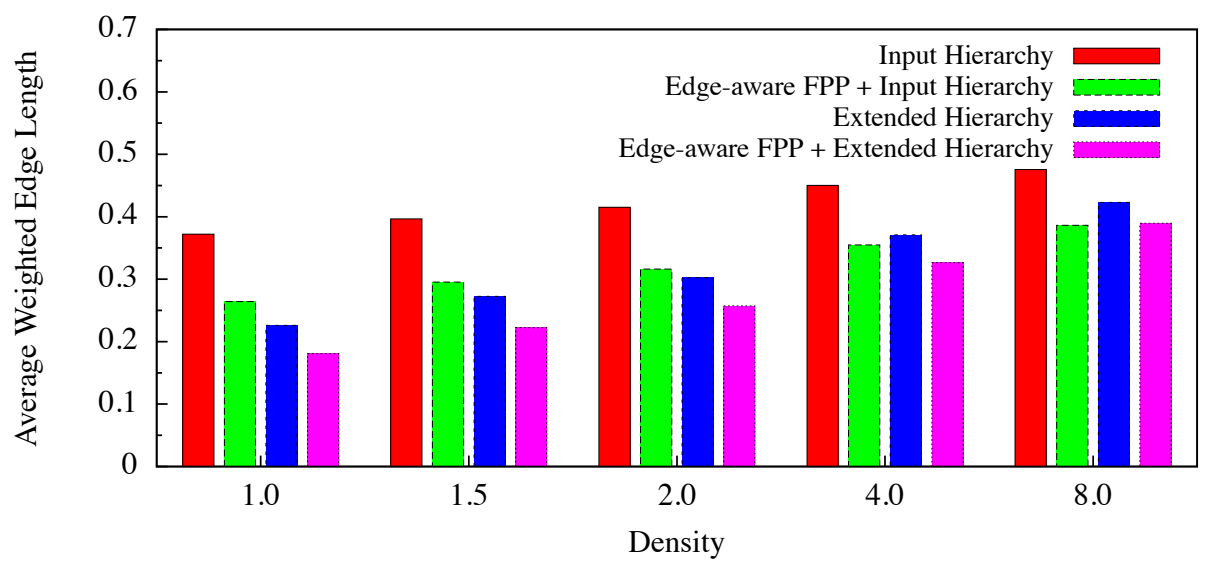

Figure 7: Comparison of the average weighted edge length.

Putting all the above steps together, we can outline the algorithm: We start with the root of the tree, assign it the boundary polygon given as input and add it to $Q$. Then we repeat the following steps until all leaves have been processed.

1. Pop a node $C$ with its boundary polygon $P$ from the queue $Q$.

2. If $C$ is a leaf, add it to the active nodes and go to the first step, otherwise $C$ must be partitioned.

3. Compute the fraction $a$ of the area required for the smaller child of $C$ and check which case applies based on $a$ and $P$.

4. Enumerate all allowed cuts and choose the cut that minimizes Equation (1).

5. Enqueue the two children in $Q$.

Notice that neither $C$ nor its children are active during the cut enumeration phase.

\section{RESULTS \& APPLICATION}

In this section, we report on an experimental evaluation of the proposed techniques to minimize edge length and introduce several domain-specific extensions.

\subsection{Evaluation of Edge Length}

In order to determine the effect on the layout, we tested our approach on real-world datasets. The instances were derived from a publicly available pyruvate kinase high-throughput screening dataset ${ }^{3}$, which has previously been used in (Wetzel et al.,

\footnotetext{
${ }^{3}$ Available from PubChem BioAssay, AID 361.
}

2009) and consists of 51,415 compounds. We generated 20 subsets by randomly selecting 200, 400, 600 and 800 compounds, respectively. For each subset we created clustered graphs according to the graph model described in Section 3 with varying densities by selecting suitable threshold parameters. We report the average weighted edge length of these instances drawn in the unit square. Figure 7 displays the results for different densities. It is clearly visible that the two presented approaches affect the edge length in a positive way. As expected, the effect is less when the density increases. Interestingly, the changes made to the fat polygon partitioning are significant even for higher densities, unlike the extension of the cluster hierarchy. The latter performs well on the sparse instances, while the result is slightly worse for the highest density in comparison to the edge-aware fat polygon partitioning. The runtime ${ }^{4}$ for the largest instance with $|V|=800$ and $|E|=6400$ is about 7s for the cluster extension and $0.6 \mathrm{~s}$ for the edge-aware fat polygon partitioning.

Figure 8 shows an example which illustrates the effectiveness of the proposed techniques. Note that in Figure 8(a) there are several long edges, some of which even cross the entire drawing area connecting nodes in the bottom left corner (cluster A) to nodes in the top right corner (cluster E). In such cases edges are bundled to avoid visual clutter. However, a downside of edge bundling is that individual edges become difficult to identify. In Figure 8(b) the same instance is drawn using the proposed optimization techniques. Here, the layout leads to short edges and individual connections are easily recognizable. Since highly similar molecules are placed close to each other, this drawing is also clearly preferable from the application point of view.

\footnotetext{
${ }^{4}$ Machine with Core i7 $2.7 \mathrm{GHz}$ and 8 GB RAM
} 


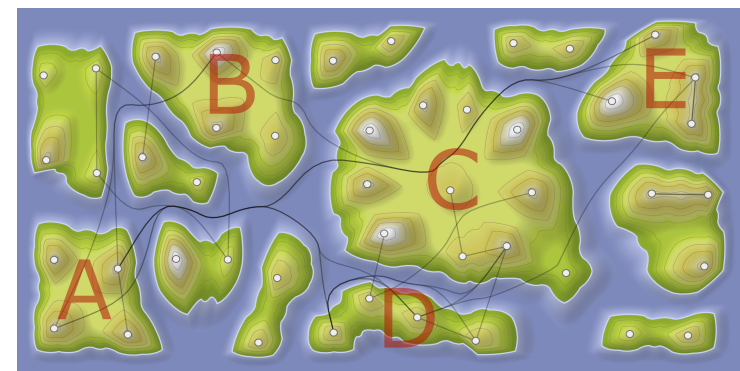

(a) Without improvements.

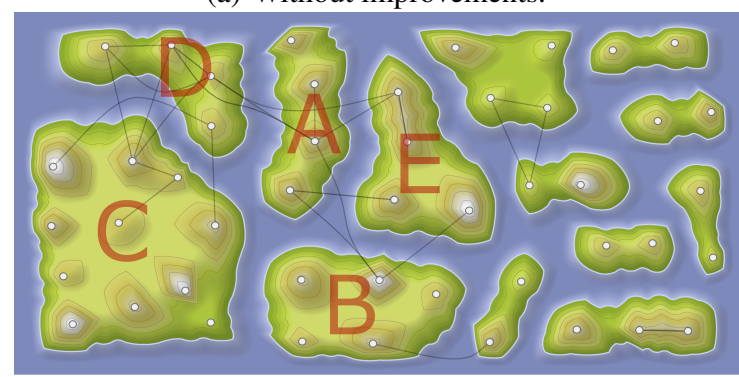

(b) Extended hierarchy and edge-aware partitioning.

Figure 8: Visual comparison of the layout generated by the original approach (a) and the proposed method (b). Letters indicate corresponding main clusters.

\subsection{Representation of Chemical Information}

In this section we report on the application of our approach to a real-world dataset and describe several extensions we incorporated to meet requirements of the application area. To focus on relevant molecules we filtered the dataset used in Section 5.1 for active compounds with $\mathrm{AC}_{50} \leq 10 \mu \mathrm{M}$ and decreasing activity direction, which yields a dataset with 432 compounds. From this subset we selected all molecules associated with scaffold tree branches containing at least 10 compounds. The threshold for the similarity graph was set to $t=0.5$. While our approach also works smoothly with larger datasets, we decided to use this small focused dataset consisting of $256 \mathrm{com}$ pounds to provide screen shots.

The resulting topographic map is shown in Figure 9(a). Since the water level is chosen such that each scaffold tree branch is represented by one island, this overview directly conveys to what extent branches are populated by compounds. With our approach the altitude represents the number of rings compounds have and whenever two molecules are located on the same hill, they share a common scaffold (according to the scaffold tree) whose number of rings is represented by the elevation level of the hill. While edges often connect molecules on the same islands, there are also edges connecting molecules placed on different is- lands (see, e.g., the central island on the left hand side and the small island south-east) indicating the presence of highly similar molecules in different branches of the scaffold tree. Placing these molecules near each other helps to generate continuous regions of highly similar molecules and is achieved by our heuristic approach. Furthermore, we support additional annotations to specifically foster the application in drug discovery.

\subsubsection{Depiction of Structural Formulae}

The most widely used representation of chemical compounds are 2D depictions of their structure. To allow a detailed investigation of structural relationships, we support displaying structural formulae. Figure 9(b) shows a close-up view of a region of an island. The shown molecules are placed on the same elevated plateau indicating their common two-ring scaffold. The two molecules on the left hand side even share a scaffold with three rings.

\subsubsection{Mapping of Properties}

While the topographic map is generated taking only structural similarity of molecules into account, relating structural features with certain properties is of utmost importance in drug discovery. We support the visualization of molecular properties by superimposing a heat map, faintly reminiscent of a temperature related weather map. Figure 9(c) shows an example where regions of highly active molecules are highlighted in red. The color gradient from red to green represents decreasing activity. Regions not colored are populated by less active molecules. The heat map is generated by computing a Delaunay triangulation and, similar to the topographic map, visualized using color encoded levels based on the property values.

\section{CONCLUSIONS \& OUTLOOK}

Organizing compound libraries by structural similarity in a way that allows for intuitive navigation for chemical tasks is challenging. We proposed a clustered graph model and adapted a technique to draw them as topographic maps. We have already received some first positive feedback from domain experts, and the representation as topographic map has been well accepted. However, a large-scale systematic evaluation of the method based on a wide range of datasets and different chemical workflows is out of the scope of this article, but an important future task.

The goal to place similar molecules near each other while respecting the cluster hierarchy leads to 


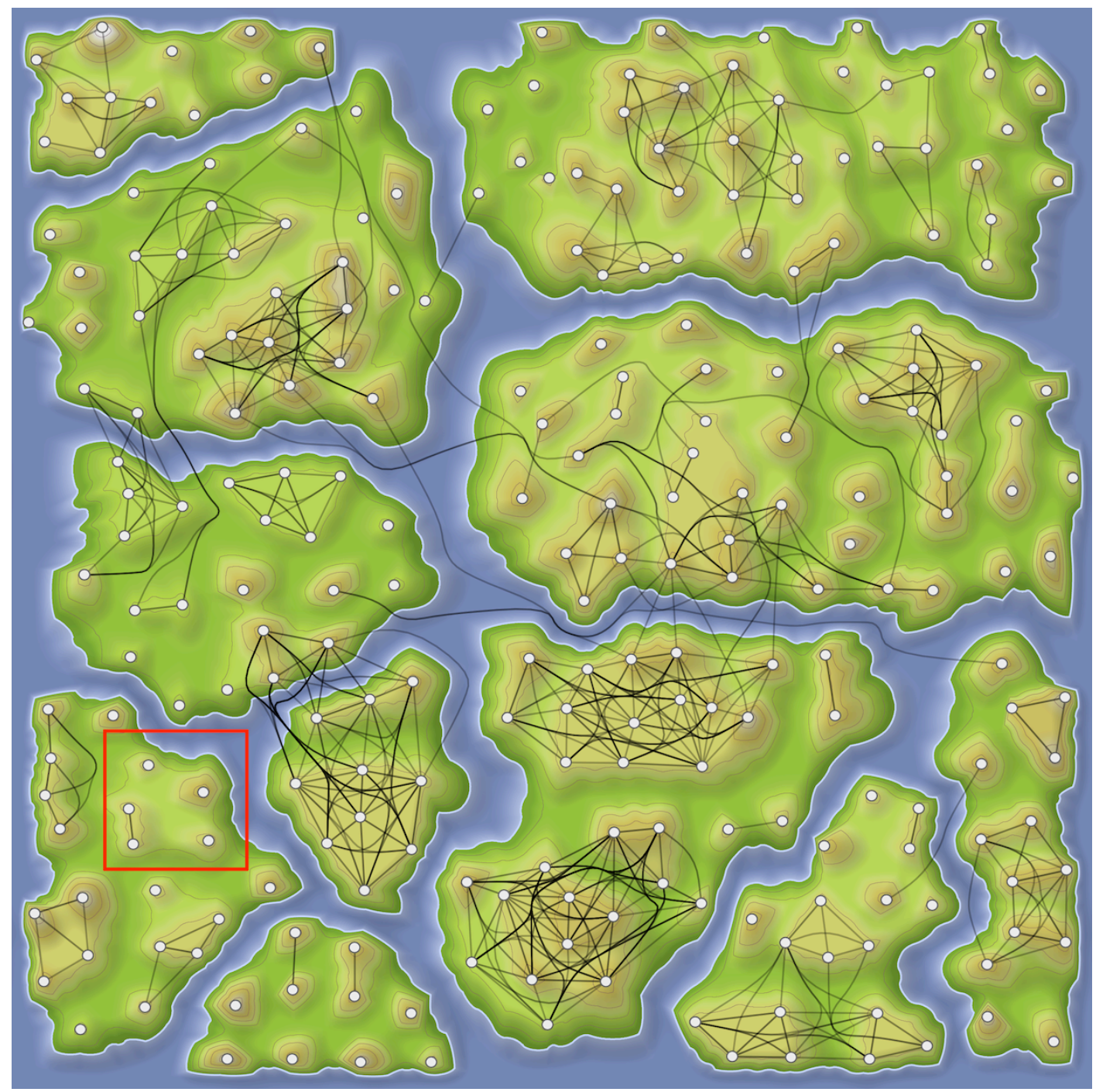

(a) Real-world instance

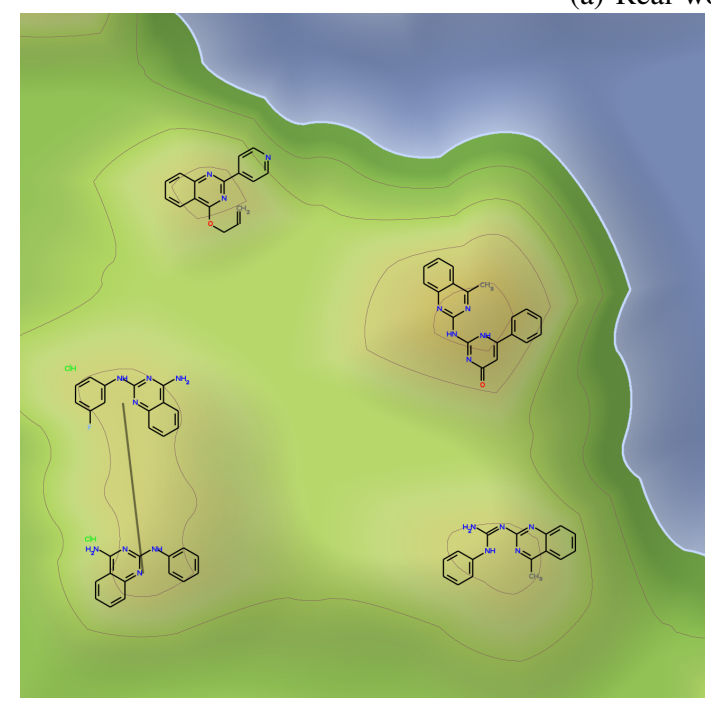

(b) Close-up view

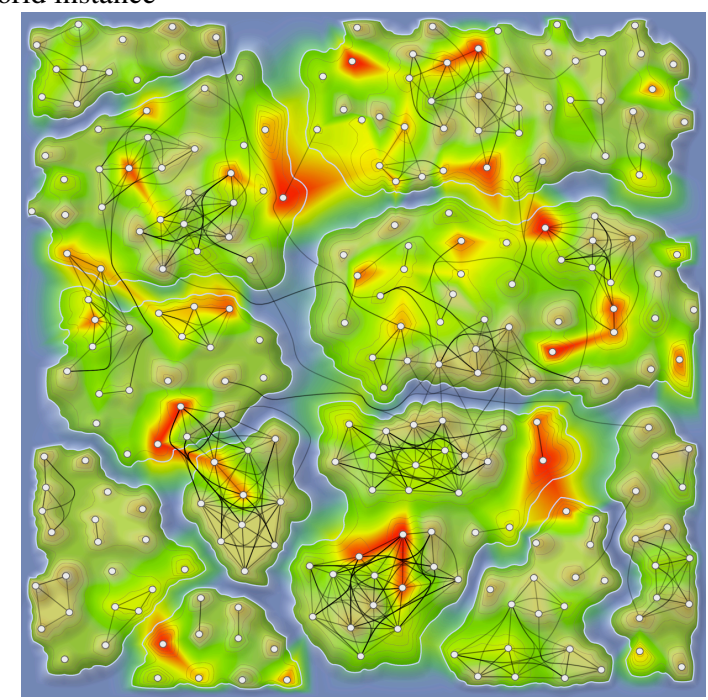

(c) Superimposed heat map

Figure 9: Visualization of the real-world instance consisting of 256 molecules (a) and the same map with property annotations (c). In (b) a close-up view of the region highlighted in red shows five molecules represented by structural formulae. 
the graph drawing problem to minimize edge length in the known approach (Gronemann and Jünger, 2012). To achieve this goal we proposed a heuristic, which exploits the degrees of freedom in the polygon partitioning procedure in order to avoid long edges. The effectiveness of the method has been demonstrated by an experimental evaluation using real-world instances.

\section{ACKNOWLEDGEMENTS}

We would like to thank Claude Ostermann and Philipp Thiel for their valuable feedback and for sharing their chemical knowledge. Research was supported by the German Research Foundation (DFG), priority programme "Algorithm Engineering" (SPP 1307).

\section{REFERENCES}

Bon, R. S. and Waldmann, H. (2010). Bioactivity-guided navigation of chemical space. Acc. Chem. Res., 43(8):1103-1114.

Brown, N. (2009). Chemoinformatics - an introduction for computer scientists. ACM Comput. Surv., 41(2).

de Berg, M., Onak, K., and Sidiropoulos, A. (2010). Fat polygonal partitions with applications to visualization and embeddings. CoRR, abs/1009.1866.

Fabrikant, S. I., Montello, D. R., and Mark, D. M. (2010). The natural landscape metaphor in information visualization: The role of commonsense geomorphology. J. Am. Soc. Inf. Sci. Technol., 61(2):253-270.

Fruchterman, T. M. J. and Reingold, E. M. (1991). Graph drawing by force-directed placement. Softw., Pract. Exper., 21(11):1129-1164.

Gansner, E. R., Hu, Y., and Kobourov, S. G. (2010). GMap: Visualizing graphs and clusters as maps. In Pacific Visualization Symposium (PacificVis), pages 201-208. IEEE.

Gansner, E. R., Hu, Y., Kobourov, S. G., and Volinsky, C. (2009). Putting recommendations on the map: visualizing clusters and relations. In Proc. ACM Conference on Recommender Systems, pages 345-348.

Girvan, M. and Newman, M. E. J. (2002). Community structure in social and biological networks. Proc. of the National Academy of Sciences, 99:7821-7826.

Gronemann, M. and Jünger, M. (2012). Drawing clustered graphs as topographic maps. In 20th International Symposium on Graph Drawing.

Herhaus, C., Karch, O., Bremm, S., and Rippmann, F. (2009). MolWind - mapping molecule spaces to geospatial worlds. Chemistry Central Journal, 3(Suppl 1):P32.

Huang, M. and Eades, P. (1998). A fully animated interactive system for clustering and navigating huge graphs.
In Graph Drawing, volume 1547 of Lecture Notes in Computer Science, pages 374-383. Springer.

Irwin, J. J. (2009). Staring off into chemical space. Nat. Chem. Biol., 5(8):536-537.

Johnson, B. and Shneiderman, B. (1991). Tree-maps: a space-filling approach to the visualization of hierarchical information structures. In Proc. of the 2nd Conference on Visualization, pages 284-291.

Klein, K., Kriege, N., and Mutzel, P. (2012). Scaffold Hunter - visual analysis of chemical compound databases. In Proc. Int. Conf. on Computer Graphics Theory and Applications and Int. Conf. on Information Visualization Theory and Applications (GRAPP \& IVAPP), pages 626-635.

Lounkine, E., Wawer, M., Wassermann, A. M., and Bajorath, J. (2010). SARANEA: a freely available program to mine structure-activity and structure-selectivity relationship information in compound data sets. $J$. Chem. Inf. Model., 50(1):68-78.

Maggiora, G. M. and Shanmugasundaram, V. (2011). Molecular similarity measures. Methods in Molecular Biology, 672:39-100.

Schuffenhauer, A., Ertl, P., Roggo, S., Wetzel, S., Koch, M. A., and Waldmann, H. (2007). The scaffold tree - visualization of the scaffold universe by hierarchical scaffold classification. J. Chem. Inf. Model., 47(1):4758.

Schuffenhauer, A. and Varin, T. (2011). Rule-based classification of chemical structures by scaffold. Molecular Informatics, 30(8):646-664.

Schulz, H.-J. (2011). Treevis.net: A tree visualization reference. IEEE Computer Graphics and Applications, 31(6):11-15.

Steinbeck, C., Hoppe, C., Kuhn, S., Floris, M., Guha, R., and Willighagen, E. L. (2006). Recent developments of the chemistry development kit (CDK) - an opensource java library for chemo- and bioinformatics. Current pharmaceutical design, 12(17):2111-2120.

Strobelt, H., Bertini, E., Braun, J., Deussen, O., Groth, U., Mayer, T. U., and Merhof, D. (2012). HiTSEE KNIME: a visualization tool for hit selection and analysis in high-throughput screening experiments for the $\mathrm{KN}$ IME platform. BMC Bioinformatics, 13 Suppl 8:S4.

Sugiyama, K. and Misue, K. (1991). Visualization of structural information: Automatic drawing of compound digraphs. IEEE Trans. SMC, 21(4):876-892.

Wawer, M., Peltason, L., Weskamp, N., Teckentrup, A., and Bajorath, J. (2008). Structure-activity relationship anatomy by network-like similarity graphs and local structure-activity relationship indices. J. Med. Chem., 51(19):6075-6084.

Wetzel, S., Klein, K., Renner, S., Rauh, D., Oprea, T. I., Mutzel, P., and Waldmann, H. (2009). Interactive exploration of chemical space with Scaffold Hunter. $\mathrm{Na}$ ture Chemical Biology, 5(8):581-583. 